Career situation of first and presenting author Student for a master or a $\mathrm{PhD}$

Introduction In rheumatoid arthritis (RA) activated neutrophils produce neutrophil extracellular traps (NETs), which provide a source of autoantigens that drives the autoimmune process. ${ }^{1}$ To identify novel immune processes that dampened neutrophil activity, we investigated a family of inhibitory glycan-binding receptors (Siglecs) that bind a specific type of glycan; called sialic acids. ${ }^{2}$ We hypothesize that sialic acid-mediated triggering of siglecs on neutrophils, which express siglec-5, -9 and -14 , will reduce their activation. In this study we focused on dampening the activity of neutrophils and thereby NET formation.

Methods Polymorphonuclear cells (PMNs) were isolated from healthy donors. Neutrophil binding of sialic acid-containing glycoconjugates was assessed by flow cytometry. Neutralizing antibodies for siglec-5/14 and -9 were used to block the interaction with the sialic acid glycoconjugates. For functional assays a branched synthetic molecule containing sialic acids (sialic acid dendrimer) was used. PMNs were rested for 1 hour at $37^{\circ} \mathrm{C}$ followed by stimulation of sialic acids dendrimers for $30 \mathrm{~min}$. Subsequently, IgA coated beads were added for $30 \mathrm{~min}$ to activate the neutrophils. NETosis was quantified via Sytoxgreen and visualised via microscopy, and phagocytosis was measured by flow cytometry.

Results Binding of sialic acid glycoconjugates was observed on neutrophils. Neutralizing siglec-5/14 and -9 receptor almost completely abolished sialic acid glycoconjugate binding to neutrophils. Neutrophils activated with IgA beads released NETs, as confirmed via microscopy. Triggering neutrophils with sialic acid dendrimer reduced this process of NETosis. The capacity to engulf IgA beads was not affected by sialic acid dendrimer stimulation.

Conclusions Neutrophils stimulated with sialic acid dendrimers show reduced activation. Patients with RA might benefit from treatment with sialic acid to dampen neutrophil-mediated autoimmune response.

\title{
REFERENCES
}

1. Wright HL, Moots RJ, Edwards SW. The multifactorial role of neutrophils in rheumatoid arthritis. Nat Rev Rheumatol 2014;10(10):593-601.

2. Macauley MS, Crocker PR, Paulson JC. Siglec-mediated regulation of immune cell function in disease. Nat Rev Immunol 2014;14(10):653-66.

Disclosure of Interest None declared.

\section{P033/031 CD38-EXPRESSING MEMORY T CELLS ARE EXPANDED IN PERIPHERAL BLOOD, CONTAINED IN INFLAMED TISSUE AND REPRESENT A POTENTIAL TREATMENT TARGET IN SYSTEMIC LUPUS ERYTHEMATOSUS}

\footnotetext{
${ }^{1,2} \mathrm{~L}$ Ostendorf*, ${ }^{2,3} \mathrm{P}$ Enghard, ${ }^{1,2} \mathrm{~F}$ Hiepe, ${ }^{1,2} \mathrm{~T}$ Alexander. ${ }^{1}$ Rheumatology, Charité Universitätsmedizin; ${ }^{2}$ Deutsches Rheumaforschungszentrum; ${ }^{3}$ Nephrology, Charité Universitätsmedizin, Berlin, Germany
}

10.1136/annrheumdis-2018-EWRR2019.25

Career situation of first and presenting author Student for a master or a $\mathrm{PhD}$.
Introduction The unresponsiveness of long-lived plasma cells (PC) to immunosuppressive and B cell depleting therapies represents a therapeutic challenge in systemic lupus erythematosus (SLE) and other immune-mediated diseases. Novel potential targets such as CD38 have emerged. ${ }^{1}$

Objectives Here, we aimed to analyze expression levels of CD38 on circulating plasmablast and peripheral blood and tissue residing lymphocyte subsets in SLE to estimate the therapeutic potential of CD38-targeting therapies.

Methods Multicolor flow cytometry was performed to investigate the CD38 expression on peripheral blood mononuclear cells (PBMCs) of SLE patients $(n=36)$, healthy controls (HC, $\mathrm{n}=20)$ and multiple myeloma $(\mathrm{MM}, \mathrm{n}=10)$ patients. In addition, kidney-infiltrating $\mathrm{T}$ cells isolated from urine were analyzed in patients with lupus nephritis (LN). To investigate the cytokine secreting potential, cytokines were analyzed intracellularly in CD38-expressing $\mathrm{T}$ cells after polyclonal stimulation in vitro with $\mathrm{PMA} /$ Ionomycin.

Results Circulating $\mathrm{CD} 19^{+} \mathrm{CD} 24^{\text {neg }} \mathrm{CD} 27^{\text {high }}$ plasmablasts are more frequent in SLE and MM patients and display higher mean fluorescence intensity for CD38 compared to HC. In SLE, CD38 is significantly higher expressed in both $\mathrm{CCR}^{+}$ central and CCR7 ${ }^{\text {neg }}$ effector memory CD4 and CD8 T cells compared to HC. Such cells co-express other markers of $\mathrm{T}$ cell activation and recent proliferative history such as HLADR and Ki-67, and are preferentially negative for FoxP3 and Helios. CD38 is most exclusively expressed on CXCR3 ${ }^{+}$ memory $\mathrm{T}$ cells isolated from urine of patients with $\mathrm{LN}$ in contrast to their $\mathrm{CXCR}^{\text {neg }}$ counterparts. Upon polyclonal stimulation, cytokine (IFN-g, IL-17, TNFa, IL-2) secreting cells were confined to memory $\mathrm{T}$ cells lacking CD38 expression.

Conclusions Expression levels of CD38 are significantly higher in peripheral blood memory B- and T-cell subsets from patients with SLE compared to HC. CD38 ${ }^{\text {pos }} \mathrm{T}$ cells co-express markers of recent activation and proliferative history, are confined to conventional memory $\mathrm{T}$ cells and contained in inflamed tissue, suggesting a pathogenic role of a chronically activated memory $\mathrm{T}$ cell compartment in SLE. The lack of effector cytokine secretion of such cells is unexpected and merits further investigation. PC depleting therapies targeting CD38 may represent a promising treatment option in SLE given their potential therapeutic effect on activated memory $\mathrm{T}$ cells in inflamed tissue.

\section{REFERENCE}

1. Hiepe F, Radbruch A. Nat Rev Nephrol 2016.

Acknowledgements We thank all study participants and blood donors.

Disclosure of Interest None declared.

\begin{tabular}{l|l}
\hline P034 & PRESENCE OF A SPECIFIC DEFECT IN M2 \\
POLARIZATION OF BLOOD MONOCYTES FROM \\
PATIENTS WITH RHEUMATOID ARTHRITIS, \\
ASSOCIATED WITH INCREASED MICRORNA-155
\end{tabular}

A Paoletti ${ }^{*}$, J Rohmer, B Ly, J Pascaud, E Riviere, G Nocturne, X Mariette. U1184 INSERM IMVA, le kremlin bicetre, France

\subsection{6/annrheumdis-2018-EWRR2019.26}

Career situation of first and presenting author Post-doctoral fellow.

Introduction Macrophages contribute in situ to the rheumatoid arthritis (RA) pathogenesis. Two distinct states of 
polarization have been recognized: the 'classically activated' (M1) and the 'alternatively activated' (M2). miRNAs are a recently discovered class of post-transcriptional regulators. Of particular relevance in RA is miR155. Its expression is upregulated in RA synovial monocytes, macrophages and fibroblasts.bIts overexpression in macrophages is associated to the production of pro-inflammatory mediators.

Objectives Here, we assessed monocytes capacity of differentiation into M2 and implication of miR155 in RA patients or controls (HD, CTD or SpA).

Methods Purified monocytes were incubated 6 day in the presence human serum $\mathrm{AB}$ (SAB) (M2). Expressions of total macrophages markers (CD11b-CD71), M2 markers (CD163, CD206, IL-10 and Arginase) and M1 markers (INOS, IFR5 and IL1 $\beta$ ) were evaluated by flow cytometry, ELISA or immunofluorescence. The microRNA transfections were performed using AMAXA technology.

Results We observed a significant decrease of macrophages induction only by SAB in RA patients as shown by a decreased expression of CD11b-CD71. We have found a specific decreased level of M2 markers. Moreover, we found ex vivo and in vitro that Adalimumab but not Etanercept or non-antiTNF drugs were able to partially correct this defect of M2

We confirmed that under SAB treatment, RA monocytes have a propensity for preferential maturation towards a proinflammatory M1-like phenotype instead of $\mathrm{M} 2$, thus contributing to synovial inflammation.

We have hypothesized the involvement of miR155 on this defect. Indeed, miR155 is induced in monocyte or macrophages and drives their inflammatory response by epigenetic regulation that lead to M1 polarization. miR155 is increased in monocytes and M2 RA compared to controls.

Subsequently we transfected monocytes from HD with miR155 mimic and we observed a decrease differentiation in M2. Conversely preliminary experiments on RA monocytes transfection with a miR-155 inhibitor allowed the restoration of M2 polarization.

Conclusions RA patients have a specific impaired maturation of monocytes to M2 as much in phenotype and in function while the differentiation to the M1 phenotype is maintained. The use of monoclonal anti-TNF restores M2 polarization defect while Etanercept or non anti-TNF drugs do not restore M2.

This lack of M2 polarization is associated with miR155 increase in RA patients that leads to IFR5/INOS expression. Preliminary experiments showed that transfection of RA monocytes with an antagomiR155 may correct this lack of M2, justifying the proof of concept trial of monocytes-targeted nanoparticles containing microRNA in CIA mouse models. Disclosure of Interest None declared.

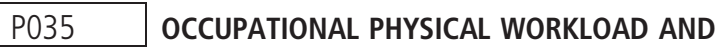 DEVELOPMENT OF ANTI-COLLAGEN TYPE II ANTIBODIES IN RHEUMATOID ARTHRITIS: RESULTS FROM THE SWEDISH EIRA POPULATION-BASED CASE- CONTROL STUDY}

\footnotetext{
${ }^{1} \mathrm{E}$ Pertsinidou*, ${ }^{2} \mathrm{P}$ Zeng, ${ }^{2,3} \mathrm{~B}$ Brynedal, ${ }^{1} \mathrm{VA}$ Manivel, ${ }^{3} \mathrm{~L}$ Klareskog, ${ }^{1} \mathrm{M}$ Mullazehi, ${ }^{2,3} \mathrm{~S}$ Saevarsdottir, ${ }^{2} \mathrm{C}$ Bengtsson, ${ }^{2,4} \mathrm{~L}$ Alfredsson, ${ }^{1} \mathrm{~J}$ Rönnelid. ${ }^{1} /$ mmunology, Genetics and Pathology, Uppsala University, Uppsala; ${ }^{2}$ Institute of Environmental Medicine, Karolinska Institute; ${ }^{3}$ Rheumatology Unit, Department of Medicine, Karolinska Institute; ${ }^{4}$ Center for Occupational and Environmental Medicine, Stockholm Country Council, Stockholm, Sweden
}

10.1136/annrheumdis-2018-EWRR2019.27
Career situation of first and presenting author Young investigator.

Introduction Separate studies have previously observed an association between exposure to physical workload (PW) and risk of developing rheumatoid arthritis (RA). Levels of antibodies against collagen type II (CII), which are associated with HLADRB $* 01$ and $* 03$ are transiently elevated at the time of RA diagnosis. PW might impose mechanical stress on the articular cartilage where CII is the main constituent, leading to immune activation and production of anti-CII antibodies.

Objectives We hypothesize that exposure to PW would associate more strongly with anti-CII positive RA than with anti-CII negative RA.

Methods Data involving 2916 incident RA cases and 5130 controls from the Swedish Epidemiological Investigations in Rheumatoid Arthritis (EIRA) population-based case-control study were analysed. Information on exposure to PW was collected through questionnaires. The odds ratios (OR) with 95\% CI of developing anti-CII positive RA or anti-CII negative RA was calculated using logistic regression.

Results The OR observed for the association between different types of PW and anti-CII positive RA ranged from $1.2(0.8-$ 1.8) to $1.7(1.5-3.1)$. The OR for the association between PW and anti-CII negative RA ranged from $1.3(1.1-1.4)$ to 1.6 (1.4-1.8). No statistically significant difference was observed between the OR for anti-CII positive RA and antiCII negative RA (all p-values $>0.10$ ). Moreover, stratification for HLA-DRB*01 and *03 did not yield any statistical differences.

Conclusions We found no evidence suggesting an association between PW and elevated anti-CII levels at the time of diagnosis.

Acknowledgements We thank the investigators and participants from the EIRA study. The authors also thank Marie-Louise Serra and Lena Nise for data collection.

Disclosure of Interest None declared.

\section{P036 B CELL NUMBER REDUCTION HAS A PREDICTIVE VALUE FOR RESPONSE TO BELIMUMAB IN A COHORT OF PATIENTS WITH SYSTEMIC LUPUS ERYTHEMATOSUS}

${ }^{1} \mathrm{~S}$ Piantoni* ${ }^{*}{ }^{1} \mathrm{~L}$ Andreoli, ${ }^{2} \mathrm{~T}$ Lowin, ${ }^{1} \mathrm{R}$ Kumar, ${ }^{1} \mathrm{~F}$ Regola, ${ }^{1} \mathrm{P}$ Airò, ${ }^{1} \mathrm{I}$ Cavazzana, ${ }^{1} \mathrm{~F}$ Franceschini, ${ }^{1} \mathrm{~A}$ Tincani, ${ }^{2} \mathrm{G}$ Pongratz. ${ }^{1}$ Rheumatology and Clinical immunology, Clinical and Experimental Sciences, ASST Spedali Civili and University of Brescia, Brescia, Italy; ${ }^{2}$ Rheumatology Department and Hiller Research Center for Rheumatology, University Hospital Düsseldorf, Düsseldorf, Germany

\subsection{6/annrheumdis-2018-EWRR2019.28}

Career situation of first and presenting author Student for a master or a $\mathrm{PhD}$.

Introduction Belimumab, the anti-B-cell activation factor (BAFF) agent, is approved for the treatment of refractory systemic lupus erythematosus (SLE). Its effect on the number of circulating B-cells is selective on naïve $(\mathrm{CD} 19+\mathrm{CD} 27-\operatorname{IgD}+)$ and immature transitional B-cells (CD19+38high24high) whose survival is strictly BAFF-dependent. On the opposite, CD27+ memory B-cells are preserved during belimumab therapy, being their maturation more related to the activation of B-cell receptor pathways.

Objectives The aim of this study is the evaluation of B-cell subpopulations in a cohort of SLE patients treated with belimumab. 